\title{
Autocognición sobre civismo y ciudadanía del estudiantado de Pedagogía en Historia, Geografía y Educación Cívica en una universidad chilena
}

Self-cognition about civism and citizenship in students of Pedagogy in History, Geography and Civic Education in a Chilean university

\section{Volumen 20, Número 1 \\ Enero - Abril \\ pp. $1-24$}

\author{
Andrés Sáez Geoffroy \\ Joel Parra-Díaz \\ Fernando Herrera Gómez
}

\section{Citar este documento según modelo APA}

Sáez Geoffroy, Andrés; Parra-Díaz, Joel y Herrera Gómez, Fernando. (2020). Autocognición sobre civismo y ciudadanía del estudiantado de Pedagogía en Historia, Geografía y Educación Cívica en una universidad chilena. Revista Actualidades Investigativas en Educación, 20(1), 1-24. Doi. 10.15517/aie.v20i1.40144 


\title{
Autocognición sobre civismo y ciudadanía, de estudiantes de pedagogía en Historia, Geografía y Educación Cívica en una universidad chilena
}

\author{
Self-cognition about civism and citizenship in students of Pedagogy in History, Geography \\ and Civic Education in a Chilean university
}

\author{
Andrés Sáez Geoffroy ${ }^{1}$ \\ Joel Parra-Díaz \\ Fernando Herrera Gómez ${ }^{3}$
}

\begin{abstract}
Resumen: Esta investigación surge por la necesidad de conocer cómo el profesorado en formación de Pedagogía en Historia, Geografía y Educación Cívica de una universidad chilena concibe elementos de civismo y ciudadanía. El objetivo de este artículo es identificar las relaciones entre la autocognición sobre civismo y ciudadanía, y el desempeño académico del profesorado en formación. Se analizan los resultados de acuerdo con el avance curricular de los sujetos de estudio, con la finalidad de identificar si el currículum incide en el desarrollo de estas competencias cívicas. La investigación se realizó durante el año 2018 bajo un enfoque cuantitativo, de corte transversal, con un diseño descriptivo y correlacional. La muestra fue de 129 sujetos y se utilizó un muestreo probabilístico estratificado. Se utilizó la escala del Estudio internacional de educación cívica y formación ciudadana del año 2009. Los resultados muestran que existe una correlación entre los puntajes en las tres categorías de la dimensión estudiada y el Promedio General Acumulado (PGA) de quienes participaron. A la vez, son inexistentes las diferencias según el avance curricular. De esta manera, se puede establecer una relación entre el desarrollo de la autocognición ciudadana y el rendimiento académico, aunque el currículum de esta carrera no favorecería el desarrollo de estas competencias. Dada la particularidad del curriculum de la carrera cursada por quienes participaron, los resultados no podrían ser generalizables. No obstante, este es un estudio de una temática poco abordada, lo que permite establecer una base sobre la cual se pueden realizar otros trabajos.
\end{abstract}

Palabras clave: estudiante universitario, educación ciudadana, historia, cognición

Abstract: This research arises from the need to know how the teaching staff of Pedagogy in History, Geography and Civic Education of a Chilean university, conceives elements of civics and citizenship. The objective of this article is to identify the relationships between selfcognition on civics and citizenship and the academic performance of teachers in training. The results are analyzed according to the curricular progress of the study subjects, with the purpose of identifying if the curriculum affects the development of these civic competences. The research was carried out in 2018 under a quantitative, cross-sectional approach, with a descriptive and correlational design. The sample was 129 subjects and a stratified probabilistic sampling was used. The scale of the International Study of Civic Education and Citizenship Training of the year 2009. The results show that there is a correlation between the scores in the three categories of the dimension studied and the Accumulative General Average (PGA) of those who participated, not existing at At the same time, differences according to the curricular progress. In this way, a relationship can be established between the development of citizen self-ignition and academic performance, although the curriculum of this career would not favor the development of these competences. Given the particularity of the curriculum of the career studied by those who participated, the results could not be generalized, however this is a study of a subject that is not very much addressed, which allows establishing a base on which other works could be carried out.

Keywords: university student, citizenship Education, History, cognition

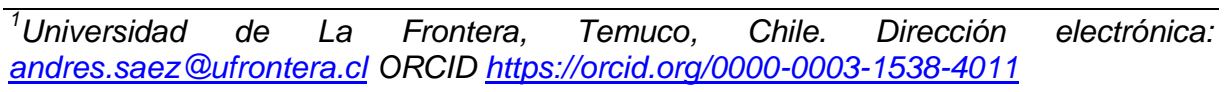

${ }^{2}$ Universidad de La Frontera, Temuco, Chile. Dirección electrónica: joel.parra@ufrontera.cl ORCID https://orcid.org/0000-0002-7119-7950

${ }^{3}$ Universidad de La Frontera, Temuco, Chile. Dirección electrónica: fernando.herrera@ufrontera.cl ORCID https://orcid.org/0000-0002-4069-3038

Artículo recibido: 22 de junio, 2019

Enviado a corrección: 24 de setiembre, 2019

Anrohado: 18 de noviemhre 201.9

Los contenidos de este artículo están bajo una licencia Creative Commons 


\section{Introducción}

Después de un amplio debate social y político en Chile, en el año 2009 se promulgó la Ley General de Educación (LGE), la cual modificaría la estructura del sistema educativo chileno dejando atrás la Ley Orgánica Constitucional de Enseñanza promulgada durante la dictadura del general Pinochet en 1989. Con este nuevo cuerpo legal se inició un camino de reformulación del sistema educativo en el que se puede destacar el diseño y promulgación de nuevas bases curriculares para la enseñanza primaria (2012), secundaria (2009, 2012, 2015 y 2019) y preescolar (2018). Esta nueva estructura curricular se vio complementada con la Ley N² 20.903 (2016), que crea el Sistema de desarrollo profesional docente, el cual establece nuevos criterios de selección para las universidades que imparten carreras de pedagogía, y crea un nuevo sistema de remuneraciones, evaluación docente y formación continua del profesorado.

En abril de 2016, la Ley $N^{\circ} 20.911$ conocida como Ley de Formación Ciudadana estableció la creación del plan de formación ciudadana para los establecimientos educacionales reconocidos por el Estado, el cual deberá identificar e incorporar, de manera transversal en el currículum de cada centro educativo, los aspectos necesarios para la preparación de ciudadanos integrales y competentes, no solo en términos de conocimientos, sino también, y fundamentalmente, en el desarrollo de habilidades y actitudes propias del ciudadano del siglo XXI. Junto con este nuevo instrumento de gestión, la Ley indica la creación de una nueva asignatura de carácter obligatoria, que las nuevas bases curriculares han definido como Educación Ciudadana para los últimos dos niveles de la educación secundaria. En el sistema educativo chileno, la formación ciudadana ha estado siempre al alero del profesorado de Historia, Geografía y Educación Cívica, y en los últimos cambios curriculares esto no ha sido la excepción.

En este escenario, el artículo busca identificar las relaciones entre la autocognición sobre civismo y ciudadanía, y el desempeño académico del profesorado en formación. Este artículo se enmarca en el proyecto IF17-0003 Evaluación de conocimientos y actitudes cívicas en la carrera de Pedagogía en Historia, Geografía y Educación Cívica de la Universidad de La Frontera, y es financiado por la Vicerrectoría de Pregrado y Vicerrectoría de Investigación y Postgrado de la Universidad de la Frontera. La Investigación fue desarrollada durante el año 2018 con el objetivo de determinar la influencia de la trayectoria curricular en los conocimientos y actitudes cívicas en la población estudiantil de la carrera 
mencionada con una proyección a incorporar mejoras y ajustes futuros a la carrera que es responsable ante el sistema educativo de la formación ciudadana.

\section{Referentes teóricos}

En las bases curriculares chilenas, la asignatura de Historia, Geografía y Ciencias Sociales se señala como uno de los ejes el desarrollo de competencias ciudadanas. El documento describe que "es fundamental que los alumnos se reconozcan como ciudadanos y desarrollen una predisposición favorable hacia la vida en comunidad, en el marco de una sociedad democrática, poniendo en práctica los valores inherentes a ello" (Ministerio de Educación de Chile [MINEDUC], 2012a, p. 181). Conceptualmente, educación cívica y formación ciudadana tienen objetivos diferentes, lo que se deriva del contexto espaciotemporal en el que se formularon. Así, Enric Prats señala que

Históricamente la idea de la educación cívica es un concepto que surgió en el siglo XIX y recorrió el siglo XX con la idea de formar ciudadanos conocedores de la institucionalidad y pertenecientes a un estado nacional, su objetivo: que el estudiantado desarrolle un patriotismo constitucional (Prats, 2012, p.3).

En contraparte, el concepto de formación ciudadana implica una amplitud temática que va más allá de las meras instituciones, lo que incorpora las nuevas formas de participación democrática y de la vida en una sociedad globalizada (Quiroz y Jaramillo, 2009; Organización de las Naciones Unidas para la Educación, la Ciencia y la Cultura [UNESCO], 2017).

Bajo este punto de vista, la educación cívica no corresponde solo a una designación antigua para la formación ciudadana, de acuerdo con elcontexto propio de donde surge, pero a nuestro juicio a pesar de que ambas designan una práctica y fin similar, la enseñanza para ser buenos ciudadanos, la formación ciudadana tiene un enfoque más amplio y centrado en múltiples aspectos, de todos modos, ambas corrientes se encuentran entrelazadas, como señalan Quiroz y Jaramillo (2009):

Indudablemente, no es una moda que gira en torno a la educación cívica y la formación ciudadana, sino más bien una resignificación de la educación cívica como una apuesta para la formación de ciudadanos; pues el contenido de ésta, la comprensión y la puesta en práctica de los mismos, no son más que elementos que dan entrada a formar ciudadanos que respondan a las exigencias de un entorno dado; es decir, se 
teje un vínculo fuerte y casi inseparable entre educación cívica y formación ciudadana. (pp. 134-135)

Lo anterior nos demuestra que la enseñanza de lo cívico-ciudadano ha sido un tema dinámico. En 1914, Dunn señalaba que la enseñanza del civismo debía contribuir sistemáticamente a entrenar ciudadanos (training of citizens). Para evaluar y calificar la efectividad de aquel adiestramiento se tendría como estándar el hecho de que cada estudiante fuera evaluado con base en el desarrollo de ciertas actitudes desde un entramado conductista. En la actualidad han variado las concepciones sobre la profesión pedagógica y, por tanto, también hay cambios en cómo se comprende la educación cívica. En este sentido, Ehrlich (1999) declara que la educación cívica es enseñarle al estudiante sobre la democracia teniendo en consideración los conceptos básicos inherentes al desarrollo histórico y los valores de dicho sistema político, pero, por sobre todo, el cómo esos saberes se transmiten mediante el trabajo con la comunidad donde se inserta el dicente. Similar es la visión de Coleman, para él la Pedagogía Cívica (Civic Pedagogies) es "darle a la juventud las competencias y virtudes que le permitan cooperar con otros ciudadanos bajo un estado de derecho democrático" (Coleman, 1998, p. 747).

Actualemente existe un relativo consenso en los currículos escolares (con excepción de los Estados Unidos, donde se insiste en el concepto de formación cívica, civic skills) de que se debe superar el antiguo modelo de la enseñanza minimalista de la ciudadanía, basado en temas relativos a la institucionalidad y constitucionalismo nacionales, para pasar a un "nuevo paradigma que se caracteriza por ir más allá de la entrega de información y que avance en la práctica y testimonio de buenas prácticas de la vida en ciudadanía" (UNESCO, 2017, p. 14).

El cambio anteriormente enunciado no solo tiene conexión con los cambios curriculares para añadir la amplitud temática de la formación ciudadana, sino también con el profesorado. En ese tópico hay una cuestión fundamental relativa a la formación inicial docente. Un informe de la UNESCO titulado La Formación Inicial Docente en Educación para la Ciudadanía en América Latina" introduce el debate que señala:

Se hace clave conocer cuáles son las oportunidades de aprendizaje que tiene el profesorado en formación. El fortalecimiento de las bases culturales de la creencia democrática, como de las capacidades de la nueva generación para la vida juntos, con cercanos (convivencia), y lejanos (ciudadanía), depende, como dicho, de manera 
importante de la experiencia escolar y ésta a su vez, en forma decisiva, de la calidad de sus docentes y su preparación. Resulta entonces de alta relevancia examinar lo que la Formación Inicial Docente lleva a cabo en la actualidad en esta trascendental área. (UNESCO, 2017, p.7)

En ese sentido, es posible indicar que, hasta el momento, la evaluación respecto a la formación cívica en el profesorado se ha centrado en los resultados del estudiantado, pero no existen estudios sobre la realidad de cómo el profesorado de pedagogía adquiere las habilidades necesarias para la enseñanza de los contenidos relativos a la formación ciudadana. En el caso de Chile y los países latinoamericanos resulta decisivo examinar la realidad de la situación, ya que el gran impacto formativo en los niños, niñas y jóvenes ocurre fundamentalmente en la enseñanza primaria y secundaria. Tanto es así que en la controvertida reforma curricular del año 2019, en Chile se pretende implementar como obligatoria la Educación Ciudadana en 3ํㅜ y 4ํaño de educación secundaria.

En esta dirección, entre los estándares que se exigen tanto a las universidades formadoras como al profesorado de la asignatura de Historia, Geografía y Ciencias Sociales se encuentra el hecho de que sean capaces de elaborar "estrategias didácticas que promuevan los aprendizajes, valores y conductas ciudadanas, conducentes a hacer de sus estudiantes sujetos activos y responsables ante el desafío de la convivencia democrática y el desarrollo sustentable" (MINEDUC, 2012, p. 162).

En esa dirección el sistema escolar chileno se ha ido adecuado y adaptando a la llegada de la formación ciudadana como tema relevante en la discusión educativa. En 2016 se promulgó la ley N²0.911 de Formación Ciudadana que asimismo señala "el Ministerio de Educación, en el marco de sus atribuciones, fomentará que en la formación inicial docente se incorpore la formación ciudadana y educación cívica" (Ley 20.911, 2016).

La dificultad ha radicado en cómo establecer espacios curriculares concretos para el desarrollo de estos procesos de enseñanza y aprendizaje. Esta es una situación en todo caso compleja, puesto que la formación ciudadana por su propia naturaleza debiera tener un espacio curricular asegurado, lo que se estaría dando con una propuesta de cambio curricular aprobado por el Consejo Nacional de Educación en mayo de 2019. En los países desarrollados, "la formación ciudadana también es una preocupación para las ciencias políticas y tiene asignadas horas en el programa de estudio" (Kedrowski, 2003, p. 225). 
En este contexto nacional, también es necesario recalcar el rol jugado por las evaluaciones internacionales en la elaboración de la política educativa. Es preciso mencionar el Estudio Internacional de Educación Cívica y Formación Ciudadana (en sus siglas en inglés ICCS) desarrollado por la Asociación Internacional para la Evaluación del Logro Educativo (IEA). En el caso de Chile, el último informe es del año 2016, y buscó evaluar las competencias ciudadanas en la población estudiantil de octavo año básico, además de aplicar un instrumento para los docentes de la asignatura responsable de la formación ciudadana. Como señala el informe "el propósito de ICCS es investigar las maneras en que los jóvenes están preparados para asumir sus roles como ciudadanos en el siglo XXI, en un conjunto de países" (ICCS, 2009, p. 11) ${ }^{3}$. El instrumento consideró tres dimensiones (1) El contenido que se evaluará (institucionalidad, normas, sistema de toma de decisiones, etc.), (2) Una dimensión afectiva conductual, relativa a las creencias sobre ciertos valores y (3) una dimensión sobre los procesos cognitivos centrada en el proceso de enseñanza-aprendizaje tanto del profesorado como del estudiantado. En nuestro caso, hemos estudiado la categoría de la percepción afectivo conductual de lo cívico-ciudadano, en especial la dimensión Auto cogniciones de los alumnos en relación con el civismo y la ciudadanía, en los futuros docentes que se integrarán al sistema escolar con altas probabilidades de ser los encargados de los planes de formación ciudadana establecidos por ley en los establecimientos.

En este sentido, es importante señalar que "las actitudes se diferencian de las creencias en que son más limitadas que éstas, pueden cambiar con el paso del tiempo y están menos arraigadas. Además, los individuos pueden tener varias actitudes contradictorias al mismo tiempo" (ICCS, 2016, p. 24). En lo particular nos interesa el factor tiempo, ya que, al llevar el estudio de esta dimensión a la formación inicial docente, es relevante evaluar el impacto que el avance curricular de una carrera de pregrado tiene en la generación de actitudes ciudadanas. De esta manera se hace necesario evaluar el impacto del avance curricular que potenciaría las actitudes cívicas, toda vez que la carrera de

${ }^{3}$ Debido a que se trata de un informe internacional de carácter colectivo y para facilitar la lectura del texto, las siguientes referencias figurarán con la sigla ICCS y su año respectivo. Las referencias son de: Schulz, Wolfram, Ainley John, Cox Cristián y Friedman, Tim (2016). Percepciones de los jóvenes acerca del gobierno, la convivencia pacífica y la diversidad en cinco países de América Latina Estudio Internacional sobre Educación Cívica y Ciudadana 2016 de la IEA Informe Lationamericano. Amsterdam, Holanda: Asociación Internacional para la Evaluación del Logro Educativo. Schulz, Wolfram, Ainley, John, Friedman, Tim y Lietz, Petra (2009). Informe latinoamericano del ICCS 2009: Actitudes y conocimientos cívicos de estudiantes de secundaria en seis países de América Latina. Amsterdam, Holanda: Asociación Internacional para la Evaluación del Logro Educativo. 
Pedagogía en Historia, Geografía y Educación Cívica ha estado orientada históricamente a la formación del profesorado que dicta la asignatura en los establecimientos escolares.

Las categorías que se asocian a las establecidas por el ICCS, que fueron analizadas en el instrumento aplicado y en este artículo, corresponden a la dimensión Auto cogniciones de los alumnos en relación con el civismo y la ciudadanía, que presenta tres subdimensiones: a) Intereses por los eventos políticos y sociales, b) Eficacia Política y c) Autoeficacia ciudadana.

\section{- Interés por eventos políticos y asuntos sociales}

EI ICCS lo define como "constructo [que] refleja la motivación de los alumnos en lo que respecta a la política" (ICCS, 2009, p. 24). Esta subdimensión permite pronosticar de manera positiva el interés por participar en asuntos políticos, pero también predice el grado de conocimiento cívico de los estudiantes (Niemi y Junn, 1998, p. 18). En el fondo a mayores grados de intereses por los contenidos más debería saber, en este caso, el profesor en formación.

El interés por informarse, participar y discutir temas ciudadanos es fundamental como punto de inicio y de motivación intrínseca para la enseñanza de la formación ciudadana. Lo crucial de este tema se debe a que antiguamente la educación cívica "se caracteriza por promover la formación de ciudadanos "informados", que se reconozcan como miembros de una comunidad nacional, a través de la transmisión de conocimientos vinculados a la historia nacional y al funcionamiento político y jurídico de las instituciones. (UNESCO, 2017, p. 14). Por ello, que los docentes en formación muestren un interés es relevante pues viene a constituir una condición sine qua non de la enseñanza de la formación ciudadana.

El punto de partida para la enseñanza de la formación ciudadana, que involucra temas de contingencia, de un currículo escolar que se amplía por la salida y/o llegada de nuevos temas obliga, que el docente responsable de la asignatura tenga un interés por estar informado en estos temas, que sea partícipe del debate público y lo lleve a las aulas. Esto es lo que permite evaluar esta categoría, la capacidad de motivación por informarse que tendrán los futuros docentes y poner en el aula escolar los temas de contingencia.

\subsection{Auto concepto en lo referente a la participación política (eficacia política)}

De acuerdo a Kenski y Jomini (2006) "political efficacy, knowledge, and participation. It has been argued that these important political variables are indicators of a properly 
functioning democracy" (p. 174). De este modo, la percepción que los futuros docentes tengan de sí mismos como sujetos políticos activos, el conocimiento y la participación, podría permitir explicar el interés en el funcionamiento de la democracia y el traspaso de dicha motivación a sus dicentes.

La participación política, que puede definirse también como Eficacia Política, puede ser entendida cómo cada ciudadano puede tomar acciones para generar un cambio en la sociedad. En este sentido, cómo los futuros docentes sienten que pueden ser parte en la generación de cambios en la comunidad cívica. Hay un sentido asociado al conocimiento en sí mismo, esto es cuánto comprenden los futuros profesores sobre los temas coyunturales y cómo son capaces de expresarlos prácticamente en la sala de clases. Por otro, como el profesorado ve la reacción del sistema político en múltiples temas.

A partir de ello se han conceptualizado dos tipos de eficacia política. Por un lado, la eficacia interna referida a la valoración personal que se tiene de determinadas acciones y por otra la eficacia externa centrada en las creencias que se tiene del sistema político (Kenski y Jomini, 2006). Por ello:

The internal dimension of efficacy refers to a person's view of his or her own capabilities in democratic politics-whether or not the individual is sufficiently informed to participate, can make good decisions, etc. In contrast, the external dimension refers to a person's view of the responsiveness of government: whether or not government is actually listening ${ }^{4}$. (Dyck y Lascher, 2009, p. 4)

Interesa sobre todo en este artículo la dimensión interna, apelando al efecto que tiene la formación inicial docente en los futuros profesores de Historia, Geografía y Educación Cívica de una universidad chilena, y si los estudios superiores expresados en el avance curricular afectan la visión personal que se tiene sobre sus propias capacidades para aportar a una sociedad democrática desde la sala de clases.

Hay, en todo caso, una diferencia fundamental entre la percepción y la práctica ciudadana efectiva, ya que "se trata de una actitud valorativa que indica la medida en que el ciudadano percibe que puede influir en el funcionamiento del sistema político" (Perea, 2001, p. 42). En la medida que el profesorado en formación presenten unos mejores indicadores de

\footnotetext{
4 "La dimensión de eficacia interna se refiere a la visión que tiene una persona de sus propias capacidades en política democrática - si el individuo está suficientemente informado o no para participar, puede tomar buenas decisiones, etc. En cambio, la dimensión externa se refiere a la visión que tiene una persona de la capacidad de respuesta del gobierno: si el gobierno está prestando atención o no". Traducción de los autores.
} 
eficacia política, debería mejorar su participación política y con ello la capacidad de intervenir directamente en los problemas de la sociedad, particularmente desde el aula.

Por último, es importante destacar "the strong relationship that social scientists have identified between young people's sense that they are competent civic actors and their desire to participate seems to provide a clear rationale for promoting positive educational experiences that can be shown to boost participants' sense of efficacy" ${ }^{5}$ (Kahne y Westheimer 2006, p. 290). Las tendencias estadísticas en esta dirección indican que la población estudiantil por el hecho de ser jóvenes, presentan una autopercepción alta respecto sus competencias de ciudadanía, lo que debería traducirse en mayores grados de participación cívica en múltiples instancias, tanto formales como informales. Sobre todo, importa la eficacia interna, en el entendido de la necesidad de la confianza docente para enseñar contenidos y habilidades a otras personas. Lo que hay que evaluar es si la eficacia crece o decrece con el avance curricular.

\subsection{Autoeficacia ciudadana}

De acuerdo al Estudio Internacional de Educación Cívica y Formación Ciudadana (en sus siglas en inglés ICCS):

Este constructo refleja la autoconfianza de los alumnos en el comportamiento de la ciudadanía activa (...) La diferencia entre el autoconcepto relativo a la participación política (eficacia política interna) y la autoeficacia ciudadana reside en lo siguiente: mientras que la eficacia política interna tiene en cuenta declaraciones globales sobre la capacidad general del alumnado para actuar políticamente, la autoeficacia ciudadana tiene en cuenta la autoconfianza del alumnado para emprender determinadas tareas en el terreno de la participación cívica (ICCS, 2009, p. 25)

Este tercer subdominio tiene relación con la capacidad de poner en práctica conocimientos y habilidades, lo que en nuestro caso se puede asociar a la puesta en marcha en el aula de una serie de estrategias didácticas.

Las consideraciones señaladas anteriormente son relevantes para el estudio, pues se constituyen en una guía para las universidades, al establecer parámetros y evaluar los

\footnotetext{
5 "la estrecha relación que los cientistas sociales han identificado entre la percepción de los jóvenes que son competentes actores cívicos y su deseo de participar, parece proporcionar un fundamento claro para la promoción de experiencias educativas positivas que pueden impulsar el sentido de eficacia de los participantes". Traducción de los autores.
} 
efectos que las estructuras curriculares de la formación inicial docente tienen sobre uno de los actores fundamentales del sistema escolar. Para sintetizar este acápite se puede señalar, por tanto, que nuestra investigación se centra en estudiar la presencia de estos tres subdimensiones enunciadas anteriormente en la formación inicial docente de una universidad chilena correlacionándolas con el avance curricular que tiene el estudiantado.

\section{Metodología}

\subsection{Enfoque}

Esta investigación cuantitativa de corte transversal se realizó utilizando un diseño descriptivo y correlacional, busca, principalmente, especificar las propiedades, las características y los perfiles de personas, grupos, comunidades, procesos, objetos o cualquier otro fenómeno que se someta a un análisis (Hernández, Fernández y Baptista, 2014). Para este estudio, el enfoque señalado es adecuado debido que queremos especificar las características, señalar los perfiles de los participantes, e investigar la relación de la autocognicíon sobre civismo y ciudadanía con el desempeño académico en las tres subdimensiones estudiadas.

\subsection{Unidades de análisis}

Las personas que participaron de esta investigación, corresponden a 129 profesores en formación que cursan estudios de pregrado, de primer a quinto año, en Pedagogía en Historia, Geografía y Educación Cívica, pertenecientes a una universidad pública ubicada al sur de Chile. La cantidad de participantes de este estudio fue determinada por la fórmula de tamaño de muestra considerando un 95\% de confianza, y un margen de error de 5\%. Esta cantidad representa al $67,18 \%$ del total de estudiantes de la carrera. Del total de la muestra, el $62,8 \%$ (81) fueron mujeres y el $37,2 \%$ (48) fueron hombres. El tipo de muestra de esta investigación fue muestreo probabilístico estratificado debido a que "es necesario segmentar la muestra en relación con estratos o categorías que se presentan en la población, y que además son relevantes para los objetivos del estudio" (Hernández, et al., 2014, p. 181). 
Tabla 1: Estratos de la muestra según año de ingreso a la carrera 2014 a 2018

\begin{tabular}{llll}
\hline Estrato & $\mathbf{N}$ & Proporción & $\boldsymbol{n}$ \\
\hline 2014 & 31 & $16,3 \%$ & 21 \\
2015 & 25 & $13,2 \%$ & 17 \\
2016 & 30 & $15,5 \%$ & 20 \\
2017 & 36 & $18,6 \%$ & 24 \\
2018 & 70 & $36,4 \%$ & 47 \\
Total & 192 & $100 \%$ & 129 \\
\hline
\end{tabular}

Fuente: Elaboración propia, 2019

Así mismo, previo a responder el cuestionario utilizado, quienes participaron del estudio aceptaron las condiciones expresadas en el consentimiento informado de acuerdo a las exigencias del comité ético científico (CEC) de la Universidad donde se realizó el estudio.

\subsection{Técnicas de recolección de información}

El instrumento utilizado en este estudio, fue digitalizado en un sistema de encuestas en línea provisto y financiado por la Universidad. Antes de aplicar el instrumento a quienes participaron del estudio, se informó al cuerpo docente de la carrera y se solicitó autorización a algunos de ellos para realizar la aplicación en alguna de las clases a estudiantes de primer a quinto año, durante el segundo semestre del año 2018.

El grupo que participó en el estudio respondió la escala del Estudio internacional de educación cívica y formación ciudadana (en inglés ICCS) que es desarrollado por la Asociación Internacional para la Evaluación del Logro Educativo (IEA).

Las categorías del ICCS analizadas en este estudio, corresponden a la dimensión Auto cogniciones de los alumnos en relación con el civismo y la ciudadanía, que contiene tres sub-dimensiones, medidas a través de una escala tipo Likert de 4 puntos. Subdimensión a) Intereses por los eventos políticos y asuntos sociales (6 ítem) y se midió con la escala "Mucho a nada", la subdimensión b) Autoconcepto en lo referente a la participación política (5 ítem) y se midió con la escala "Muy en desacuerdo a muy de acuerdo" y la subdimensión c) Autoeficacia ciudadana (7 ítem) y se midió con la escala "Muy bien a mal".

\subsection{Análisis y procesamiento de datos}


En primera instancia se efectuaron análisis estadísticos con la finalidad de calcular la confiabilidad de la escala en las subdimensiones de "Intereses por los eventos políticos y asuntos sociales", "Autoconcepto en lo referente a la participación política" y "Autoeficacia ciudadana", analizando las medias de cada una de las subdimeniones. A continuación, se calculó la Correlación de Pearson de estas tres variables con el PGA del nivel cursado por el estudiantado. De manera complementaria y para finalizar estos análisis, se realizó la prueba Anova de un factor, para detectar posibles diferencias de los resultados según el año de estudio cursado. Para efectuar estos análisis se utilizó el software SPSS en su versión 25.

\section{Resultados}

Respecto a la distribución de los datos, la asimetría fue negativa $(-, 058)$, y la curtosis platicúrtica (-,291), la media es de 1,98 y la desviación estándar es igual a ,432.

A continuación, se describen los resultados de la confiabilidad de la escala, la correlación de las tres subdimensiones con el Promedio General Acumulado (PGA) ${ }^{6}$ de cada año o nivel cursado, y las posibles diferencias en los resultados según el avance curricular.

\subsection{Confiabilidad y validez de la escala}

La confiabilidad, se estimó a través del método de Consistencia Interna (Hogan, 2004). Así, la subdimensión de "intereses por los eventos políticos y asuntos sociales" muestra un nivel de confiabilidad (Alpha de Cronbach) de 0,816; la subdimensión de "Autoconcepto en lo referente a la participación política" de 0,833, mientras que la subdimensión "Autoeficacia ciudadana" tiene una confiabilidad de 0,848. Finalmente, la confiabilidad general de las tres subdimensiones es de 0,900 , con lo que se puede señalar que la confiabilidad de la escala es alta.

Respecto a la validez y en consideración del propósito de la investigación, se presentan los resultados de la validez de contenido y constructo del instrumento.

Validez de contenido: dado que las preguntas utilizadas se basan en el instrumento ICCS que forman parte de una investigación internacional, se asume la pertinencia conceptual de las mismas.

Para la validez de constructo se utilizó el método de validez convergente y discriminante recomendado por Campbell y Fiske (1959). Del análisis se desprende que al

\footnotetext{
${ }^{6}$ Corresponde al promedio de notas del estudiante según su avance curricular en una carrera de pregrado.
} 
comparar el promedio de las subdimensiones con las preguntas que conforman cada una de las subdimensiones, todas presentan correlaciones superiores a 0,477 a excepción de la pregunta $\mathrm{N}^{\circ} 18(0,147)$ perteneciente a la subdimensión autoeficacia ciudadana.

Respecto a la validez discriminante, al calcular las correlaciones entre las subdimensiones y los ítems que corresponden a las otras subdimensiones, todos presentan valores inferiores a 0,229 lo que da cuenta de la pertinencia de los ítems al ser comparados con subdimensiones que no se relacionan directamente al fenómeno que se está midiendo.

En consideración de lo anterior, es posible concluir que el instrumento utilizado posee los parámetros con validez y confiabilidad necesarios para el uso que se le da en la presente investigación.

\subsection{Correlaciones bivariadas entre el desempeño académico del curso o nivel y subdimensiones de la autocognición ciudadana}

La variable desempeño corresponde al PGA del estudiantado en las distintas asignaturas cursadas hasta el primer semestre del año 2018. El PGA por nivel se señala en la Tabla 2. Las correlaciones entre las tres subdimensiones estudiadas y el PGA se presentan en la Tabla 3, las que serán analizadas en detalle en los resultados del artículo.

Tabla 2: Promedio general acumulado (PGA)

según año que cursa de la carrera del profesorado en formación en una universidad chilena.

\begin{tabular}{ccc}
\hline Cohorte & Año que cursa & PGA \\
\hline 2014 o & $5^{\circ}$ año & 4,84 \\
antes & & \\
2015 & $4^{\circ}$ año & 5,17 \\
2016 & 3ํ año $^{\circ}$ & 4,74 \\
2017 & $2^{\circ}$ año & 4,83 \\
2018 & 1 año & 4,93 \\
\hline
\end{tabular}

Fuente: Elaboración propia, 2019 
Tabla 3: Correlación entre Intereses por los eventos políticos y asuntos sociales, Autoconcepto en lo referente a la participación política, Autoeficacia ciudadana, y desempeño académico (PGA).

\begin{tabular}{lccc}
\hline \multicolumn{1}{c}{ Subdimensión } & $\begin{array}{c}\text { Autoconcepto en lo } \\
\text { referente a la } \\
\text { participación política }\end{array}$ & $\begin{array}{c}\text { Autoeficacia } \\
\text { ciudadana }\end{array}$ & PGA \\
\hline $\begin{array}{l}\text { Intereses por los eventos } \\
\text { políticos y asuntos sociales }\end{array}$ & $0,533^{*}$ & $0,466^{*}$ & $0,163^{*}$ \\
$\begin{array}{l}\text { Autoconcepto en lo referente a } \\
\text { la participación política }\end{array}$ & & 0,609 & $0,558^{*}$ \\
Autoeficacia ciudadana & & & $0,408^{*}$ \\
\hline
\end{tabular}

* La correlación es significativa al nivel 0,01 (bilateral)

Fuente: Elaboración propia, 2019

Por otra parte, dado el nivel ordinal de la variable años de estudio, mediante Anova de un factor se exploró si los promedios de autocogniciones de alumnos en relación al civismo y la ciudadanía tendían a aumentar o disminuir conforme aumentaban los años de estudio.

Para utilizar este estadístico se procedió a revisar los supuestos de Anova. De esta manera la homogeneidad de la varianza de cada subdimensión se revisó con el test de Levene ( $p$-valor > 0,05), dando como resultado en la subdimensión Intereses por los eventos políticos y asuntos sociales $(p=0,071)$, en la subdimensión autoconcepto en lo referente a la participación política $(p=0,239)$, y en la subdimensión autoeficacia ciudadana $(p=0,214)$, cumpliendose con estos resultados este supuesto de Anova.

Por otra parte, el supuesto de normalidad, y producto de la cantidad de datos disponibles, se calculó con el estadístico Kolmogorov Smirnof (KS). De esta manera, el cálculo en las tres subdimensiones, y considerando los cinco años que cursan el profesorado en formación participantes del estudio, dio p-valor $>0,05$, lo que indica que se cumple el supuesto de normalidad.

Como puede verse en la Tabla 4, no se aprecia una tendencia ascendente y significativa en los resultados de la Anova, por tanto, se podría señalar que en la medida que el estudiantado avanza en el plan curricular, ninguna de los tres subdimensiones ni en el total de estas dimensiones se incrementan o desarrollan, no existiendo diferencias en los puntajes de las distintas cohortes (puntaje F). 
Tabla 4: Comparación entre años de ingreso a la carrera y subdimensiones del cuestionario.

\begin{tabular}{|c|c|c|c|c|c|c|}
\hline Subdimensión & $\begin{array}{l}2018 \\
(n=47)\end{array}$ & $\begin{array}{l}2017 \\
(n=24)\end{array}$ & $\begin{array}{l}2016 \\
(n=20)\end{array}$ & $\begin{array}{l}2015 \\
(n=17)\end{array}$ & $\begin{array}{l}2014 \\
(n=21)\end{array}$ & $\mathbf{F}$ \\
\hline \multicolumn{7}{|c|}{ Intereses por los eventos políticos } \\
\hline \multicolumn{7}{|c|}{ y asuntos sociales } \\
\hline M & 1,73 & 1,79 & 1,82 & 1,62 & 1,81 & 0,500 \\
\hline DE & 0,589 & 0,509 & 0,437 & 0,376 & 0,409 & \\
\hline \multicolumn{7}{|c|}{ Autoconcepto en lo referente a la } \\
\hline \multicolumn{7}{|c|}{ participación política } \\
\hline M & 1,95 & 1,93 & 2,04 & 1,94 & 2,05 & 0,281 \\
\hline DE & 0,587 & 0,466 & 0,413 & 0,429 & 0,463 & \\
\hline \multicolumn{7}{|c|}{ Autoeficacia ciudadana } \\
\hline M & 2,16 & 2,04 & 2,37 & 2,12 & 2,18 & 0,975 \\
\hline DE & 0,691 & 0,483 & 0,500 & 0,402 & 0,503 & \\
\hline \multicolumn{7}{|c|}{ Total subdimensiones } \\
\hline M & 1,94 & 1,92 & 2,10 & 1,90 & 2,05 & 0,821 \\
\hline DE & 0,517 & 0,414 & 0,402 & 0,278 & 0,377 & \\
\hline
\end{tabular}

Fuente: Elaboración propia, 2019

La Tabla 5 muestra una síntesis con algunos datos descriptivos de las tres subdimensiones y el promedio de la escala total.

Tabla 5: Síntesis de los resultados según subdimensión contenida en el cuestionario.

\begin{tabular}{lccccc}
\hline Subdimensión & $\boldsymbol{n}$ & Mínimo & Máximo & Media & $\begin{array}{c}\text { Desviación } \\
\text { estándar }\end{array}$ \\
\hline $\begin{array}{l}\text { Intereses por los eventos } \\
\text { políticos y asuntos sociales }\end{array}$ & 129 & 1 & 3 & 1,75 & 0,498 \\
$\begin{array}{l}\text { Autoconcepto en lo referente a } \\
\text { la participación política }\end{array}$ & 129 & 1 & 3 & 1,98 & 0,497 \\
$\begin{array}{l}\text { Autoeficacia ciudadana } \\
\text { Total escala }\end{array}$ & 129 & 1 & 4 & 2,16 & 0,563 \\
\hline
\end{tabular}


Fuente: Elaboración propia, 2019

\section{Discusión de los resultados}

El profesorado de Historia, Geografía y Ciencias Sociales de Chile se ha "hecho cargo de asumir estos espacios formativos (...) no solo porque tradicionalmente han sido considerados los más idóneos para esta tarea, sino además porque en las propuestas curriculares, la formación ciudadana es uno de los objetivos fundamentales de la enseñanza de la Historia y las Ciencias Sociales" (García, 2016, p. 66). Dicha idoneidad proviene en principio de la existencia de una trayectoria curricular formativa del profesorado que, de manera directa e indirecta, recurrente o concurrente ha incorporado dichas temáticas a partir de rediseños en su estructura curricular como muy bien lo han apuntado una serie de estudios (Cox, Mardones, Farías y García, 2014; UNESCO, 2017). La carrera y el estudiantado mencionado en este artículo no son la excepción.

Por el momento, la mayoría de los estudios sobre la formación inicial del profesorado en educación ciudadana, se han centrado en comparar el currículum escolar prescrito y su evolución o bien en el discurso de los actores del proceso educativo (profesorado y estudiantes). Para el caso de España, pero que también es generalizable a Iberoamérica, se señala en un estudio reciente que "aunque en las pasadas décadas se ha escrito en abundancia acerca de la educación para la ciudadanía, al menos en España se sabe muy poco acerca de cómo se educa para educar en la ciudadanía en las instancias de formación del profesorado" (Estelles, 2017, p. 39).

En términos generales los resultados de la aplicación del cuestionario a profesores en formación, podemos concluir que los puntajes han sido altos en todas las subdimensiones como se puede observar en la Tabla 5. Respecto del total de subdimensiones la media es alta, hay que considerar que los puntajes mientras más cercanos a 1 son más altos y los más cercanos a 4 más bajos. En el caso de la subdimensión de intereses por los eventos políticos y asuntos sociales tiene una media general de 1,75, esto significa que en general los futuros profesores presentan un alto interés por los temas atingentes a la formación ciudadana. En segundo lugar, la media del autoconcepto en lo referentes a la participación política es de 1,98 por lo que la eficacia política se encuentra en términos intermedios. Por último, la tercera subdimensión de autoeficacia ciudadana $(2,16)$, presenta unas medias orientadas a 
niveles bajos de capacidad de influir en los procesos políticos. A continuación, realizaremos un análisis detallado de estos aspectos.

Como se señaló, los intereses por eventos políticos y asuntos sociales presentan medias que van desde el 1,62 al 1,73, se trata de puntajes que están cercanos al punto óptimo de interesarse por materias políticas. Del mismo modo se trata de valores que están relativamente estables en las cohortes de ingreso por lo que se podría presuponer que la estructura curricular no estaría influyendo en incentivar el interés por temas de índole política, sino que más bien se trataría de un interés innato provocado por la automotivación al respecto, explicable por los elevados índices de autoeficacia política.

Al respecto hay que considerar la premisa sostenida por un estudio de los Estados Unidos que, a modo de recomendación a las instituciones públicas, considera que, si los profesores son capaces de acercarse y comprender mejor los temas políticos, esto debería traducirse en una mejor enseñanza de la formación ciudadana-cívica (Niemi y Junn, 1998, p. 158). En nuestro caso, producto de los buenos resultados sobre el interés del grupo de estudiantes, podría afirmarse que existe una propensión a que los titulados de la Carrera de Pedagogía en Historia, Geografía y Educación Cívica de esta Universidad pudieran desarrollar una buena enseñanza de estos contenidos, pero el problema identificado tiene una relación con el aporte que realiza la estructura curricular de la institución formativa en la motivación intrínseca del futuro docente, ya que no es posible observar diferencias significativas entre los niveles de avance curricular en los que se encuentra el profesorado en formación.

El caso del autoconcepto en lo referente a la participación política nos permitirá enfatizar aún más lo anterior. La situación de los puntajes distribuidos por los niveles formativos no expresa un patrón, lo que es similar a la subdimensión anterior, los puntajes están en el orden de 1,94 a 2,05, esto es cercanos a los valores medios y de manera uniforme en todas las cohortes. En términos de la eficacia interna sabemos que "esta tiene un efecto directo positivo sobre la participación política, la que también se expresa en la disposición general por participar" (Amnå, Munck y Zetterberg, 2004, p. 17).

Estaríamos perfilando entonces unos dicentes que demuestran un alto interés por temas político-sociales, pero a la vez, no se perciben mayores habilidades de desempeño cívico-ciudadano. Sabemos que las investigaciones que relacionan la autoeficacia con la participación en la juventud, dan cuenta de la importancia de la participación como fuente de interacciones que permite el desarrollo de habilidades de los y las jóvenes, permite la 
acumulación de capital social y, por lo tanto, favorece la proyección de los jóvenes como ciudadanos activos en sus comunidades (Velásquez, Martínez y Cumsille, 2014, p. 89). Dicha situación tendría que ver con un asunto contextual contingente, relacionado con la cada vez mayor abstención de participación en los diferentes procesos políticos. Hemos dicho en el marco teórico que la eficacia política es un indicador elemental para explicar el funcionamiento del sistema democrático (Kenski y Jomini, 2006, p. 174), por tanto, una explicación dable a este tópico sería el desinterés de los ciudadanos en el mismo sistema institucional político.

La tercera subdimensión del presente estudio, autoeficacia ciudadana, es la que puntúa más bajo de las 3 analizadas, con medias sobre los 2 puntos, y que van del 2,04 al 2,37, y que del mismo modo no muestra diferencias significativas entre las diferentes cohortes. Si bien existe una alta valoración en relación a la autoeficacia política y al interés por eventos públicos y asuntos sociales, las personas participantes no perciben en igual nivel su capacidad de poner en práctica estos conocimientos y saberes cívico-políticos. Si bien los aprenden en la universidad, estas experiencias de aprendizaje no están intencionadas para una futura pedagogía, por tanto carecerían de lo que Shulman definió como conocimiento didáctico del contenido (UNESCO, 2017; Shulman, 2005)

Esto conlleva la necesidad de que el proceso formativo promueva una ciudadanía activa, ya que es posible que las estructuras curriculares no estén produciendo un fuerte compromiso cívico en el centro de la experiencia de aprendizaje. Este punto es interesante ya que según Schugurensky y Myers (2013) esta cuestión ayuda en el desarrollo de mejores profesores dispuestos y capaces a enseñar los fundamentos de la ciudadanía a través de procesos democráticos (p. 162).

Al momento de correlacionar las diferentes dimensiones con el PGA pueden establecerse una serie de relaciones que permiten ahondar aún más alguno de los tópicos planteados. En el caso del interés por los eventos políticos y asuntos sociales presentan una correlación alta con un valor de 0,533, de acuerdo a Kahne y Westheimer (2006) la relación estrecha que se ha identificado en la población joven como actores cívicos competentes (autoconcepto) y su deseo por participar (interés), son factores claves para promover experiencias educativas positivas que realcen el sentimiento de eficacia política. En este sentido las correlaciones para los aspectos mencionados permiten concluir que los futuros profesores de Historia, Geografía y Educación Cívica se perciban como los más idóneos para la enseñanza del contenido. Lo anterior produce una paradoja en el tema de la 
idoneidad, ya que como hemos mencionado pareciera ser que tiene un mayor peso el interés y la motivación personal por sobre la que pudiera impulsar la estructura curricular, lo que es explicado por una correlación baja -a pesar de ser significativa- con el indicador de rendimiento académico (PGA) con un valor de 0,163. En esa dirección se encuentran también los resultados del estudio de García (2016) respecto a la autoimagen profesional del profesorado de Historia y Ciencias Sociales como responsables de la formación ciudadana en el aula.

La alta correlación entre el autoconcepto de participación (eficacia política) con Autoeficacia ciudadana $(0,609)$, permite señalar que los futuros docentes tienen una alta participación política, por eso la correlación es elevada, de hecho, es la más elevada de todas las calculadas como se puede observar en la Tabla 3. Si sumamos a este resultado el alto interés por tópicos político-sociales se podría señalar que los futuros docentes buscan la transformación a partir del rol social que pretenden cumplir en el futuro, lo que guardará una relación especial con la motivación intrínseca que hemos logrado identificar.

En esa línea nos interesa una correlación entre la eficacia política (autoconcepto) con el avance curricular (PGA), que se encuentran en un valor de 0,558, una correlación alta y significativa que nos permite presuponer dos cosas. La primera es que el autoconcepto de participación política y la eficacia se ve influida por el rendimiento académico, por ello un mayor rendimiento académico repercutiría en una mayor eficacia política, y viceversa, aquellos puntajes descendidos no. En segundo lugar, es que esta situación se ve refrendada por otra correlación en ese sentido, la correlación entre la autoeficacia ciudadana y el PGA de 0,408 que es igualmente significativa. Por tanto, más que la trayectoria curricular sería el rendimiento académico, influenciado por factores extrínsecos e intrínsecos, el posible factor explicativo de mayores grados de eficacia ciudadana y política.

Al respecto podemos establecer que en la medida que el profesorado en formación avanza en su trayectoria curricular, este avance no es significativo ya que se mantiene estable en todas las cohortes lo que evidenciaría un escaso impacto formativo de la estructura curricular. Esto de todos modos es sintomático con la condición de la FID sobre la formación del profesorado del área, en un estudio reciente, se ha sostenido que "con respecto a la cantidad de tiempo dedicado a la formación ciudadana, los académicos que participaron de las entrevistas, estiman que este factor constituye una de las principales debilidades de la formación ciudadana incluida en los programas de formación inicial" (Cox et al., 2014, p. 40). De este modo la estructura curricular que se evaluó con el cuestionario 
responde a un modo latinoamericano de abordar la formación ciudadana, con pocos cursos y cuya vinculación transversal no estaba del todo claro, por lo que los resultados demuestran una generalidad y no una excepción en el concierto de nuestro de continente.

Producto de estos resultados la carrera de Pedagogía en Historia, Geografía y Educación Cívica ha impulsado un proceso de revisión que involucra entre otras medidas, la instalación de nuevas asignaturas que motiven en el estudiantado un aumento de la eficacia política y la autoeficacia ciudadana, pero también a un rediseño de los programas de asignaturas relativas a la temática que incluya didáctica y otras formas que permitan transformar el interés del profesorado en formación en agentes ciudadanos activos en la formación de sus dicentes. Se espera por tanto en un futuro estudio, comparar los resultados para determinar si ha existido una variación en ellos y si efectivamente las estructuras curriculares están acordes a las nuevas exigencias del medio escolar.

\section{Conclusiones}

A nivel mundial la formación cívica ha ido dando paso a la formación ciudadana como forma de educar a los niños, niñas, jóvenes y adolescentes para la vida de una comunidad de carácter global. Estos cambios han repercutido a múltiples escalas. En un nivel global por la preocupación de incluir estos elementos de enseñanza en el curriculum escolar y universitarios. A nivel estatal por medio de una serie de reformas destinadas a incluir la formación ciudadana en la estructura de los sistemas educativos nacionales. Mientras en el nivel de la gestión universitaria, en lo que nos interesa que es la formación del profesorado los cambios de los contenidos y temas de enseñanza obligan el desarrollo de adaptaciones curriculares que permitan que los futuros docentes tengan las habilidades para enfrentarse a este desafío. En este artículo se ha descrito el impacto de todas estas dimensiones en una microescala, en la formación del profesorado de una universidad chilena que ha tomado acciones para adaptarse al nuevo contexto educativo. Como lo han demostrado los resultados no pretendemos un resultado generalizable, por dos motivos, el primero es porque este es el primer estudio que se hace sobre profesores en formación; en segundo lugar, porque corresponde solamente a una universidad chilena. De ello se desprende la necesidad en el futuro de poder enlazar estos resultados con los asociados a otros centros educativos y realidades formativas, pero constituye sin duda un primer paso necesario.

Entre las principales conclusiones que se puedan señalar es posible destacar que el avance curricular de los estudiantes de la carrera estudiada no determina la eficacia política 
de los futuros docentes. Esta una cuestión relevante por que los cambios curriculares que se han visto obligadas a realizar las instituciones formativas de docentes han tenido como objetivo generar efecto sobre la formación del estudiantado. De todos modos, estos resultados deben ser tomados con cautela ya que implican a generaciones de estudiantes que se ha vistos afectados por estos cambios, habrá en el futuro que volver a aplicar el instrumento para comparar si el avance curricular de las nuevas generaciones tiene un efecto en la eficacia política y con ello si los cambios curriculares, sea nivel estructural, metodológico y didáctico son los necesarios para la formación del profesorado. Este estudio en ese sentido lo que nos demuestra es que el modelo formativo anterior, más orientado a la educación cívica, no tenía un impacto directo en la eficacia política de los futuros docentes cuando la pretensión es que así fuera.

Otra de las conclusiones destacables es el hecho de que la eficacia política se ve mediatizada por la motivación que presentan los estudiantes sobre los temas políticos y relativos a la formación ciudadana. Por lo que también se trataría de condiciones de entrada al profesorado necesarias para que el futuro docente de historia, geografía y educación cívica pueda desempeñarse en el contexto de la formación ciudadana. De todo esto resultada que tanto la trayectoria del curriculum universitario, así como la motivación por el aprendizaje son temas claves para formar al profesorado. La motivación permitiría propiciar un desarrollo activo de la ciudadanía que se traduciría en la aplicación de mejores estrategias didácticas de enseñanza.

En definitiva, lo exploratorio del trabajo asociado a una carrera particular de una universidad chilena permite definir la necesidad de reevaluar constantemente el impacto de la trayectoria curricular en los intereses de temas políticos y en la eficacia política, para valorar si cabe señalar un patrón formativo o bien si los cambios curriculares son adecuados para la formación del profesorado que enseñará ciudadanía en los establecimientos escolares. Anticiparnos a los nudos críticos de la formación del profesorado permitiría tomar decisiones informadas en los cambios y ajustes curriculares que no este solamente dictadas por la legislación, sino que también por la naturaleza propia de los estudiantes.

Los alcances de este estudio en ese sentido están limitados por la escala geográfica de aplicación del instrumento, cabría desarrollar en el futuro nuevos estudios, tanto en la misma universidad, como en otras de Chile y Latinoamérica, que nos permitan definir un perfil del profesorado responsable de la formación ciudadana en las aulas escolares e ir evaluando el proceso formativo, no solo el final del mismo, tomando las acciones adecuadas para mejorar 
la instrucción de los dicentes. Todo ello repercutirá en una mejora en la calidad de la enseñanza de la formación ciudadana en las aulas, objetivo prioritario en la política educativa mundial, nacional y local.

\section{Agradecimientos}

A la Vicerrectoría de Pregrado y la Dirección de Investigación de la Vicerrectoría de Investigación y Posgrados de la Universidad de La Frontera. Proyecto de investigación Formativa IF-17-0003.

\section{Referencias}

Amnå, Erik, Munck Ingrid y Zetterberg Pär. (2004). Meaningful Participation? Political Efficacy of Adolescents in 24 countries. Emerging Repertoires of Political Action: Toward a Systematic Study of Postconventional Forms of Participation Joint Sessions of Workshops. Uppsala, Suecia: ECPR.

Campbell, Donald y Fiske, Donald. (1959). Convergent and discriminant validation by the multitrait-multimethod matrix. Psychol Bull, (56), 81-105.

Coleman, Joe. (1998). Civic pedagogies and liberal-democratic curricula. Ethics, 108(4), 746761. doi: $10.1086 / 233850$

Cox, Cristian, Mardones, Rodrigo, Farías, Ana y García, Carolina. (2014). Currículos comparados, percepciones docentes y formación de profesores para la formación ciudadana: tendencias y proposiciones de mejoramiento. Concurso políticas públicas, propuestas para Chile. Santiago, Chile: Pontificia Universidad Católica.

Dudley, Robert y Gitelson, Alan (2003). Civic education, civic engagement, and youth civic development. Political Science and Politics, 36(2), 263-267. doi: $10.1017 /$ S1049096503002191

Dunn, Arthur. (1914). By what standards shall we judge the value of civic education?. The Journal of Education, 80(3), 76-77.

Dyck Joshua y Lascher Jr. Edward L. (2009). Direct democracy and political efficacy reconsidered. Political Behavior, 31(3), 401-427. doi: 10.1007/s11109-008-9081-x

Ehrlich, Thomas (1999). Civic education: lessons learned. Political Science and Politics, 32(2), 245-250. doi: 10.2307/420559

Estelles, Marta (2017). Imágenes sobre la educación para la ciudadanía en la formación inicial del profesorado. REIDICS: Revista de Investigación en Didáctica de las Ciencias Sociales, $1, \quad 39-53.10$ Recuperado de https://dialnet.unirioja.es/servlet/articulo?codigo=6194080 
García, Carolina (2016) Interpretaciones y paradojas de la educación ciudadana en Chile Una aproximación comprensiva desde las significaciones ciudadanas y pedagógicas de los profesores de historia (Tesis de Magíster). Pontificia Universidad Católica, Santiago, Chile. Recuperado de http://repositorio.conicyt.cl/bitstream/handle/10533/182493/GARCIA_CAROLINA_2864 D.pdf?sequence=1

Hernández, R., Fernández, C., y Baptista, P. (2014). Metodología de la investigación (6ª ed.). México: Mc Graw-Hill.

Hogan, T. (2004). Pruebas Psicológicas. México: El manual moderno.

Kahne, Joseph y Westheimer, Joel (2006). The Limits of efficacy educating citizens for a democratic society. En Giarelli, James y Rubin, Beth, Social studies for a new Millennium: re-envisioning civic education for a changing world. Mahwah, Nueva Jersey, Estados Unidos: Lawrence Erlbaum. Recuperado de http://www.democraticdialogue.com/DDpdfs/Efficacy_WestheimerKahne.pdf

Kenski, Kate y Jomini, Natalie (2006). Connections between internet use and political efficacy, knowledge and participation. Journal of Broadcasting \& Electronic, 50(2), 173192. http://citeseerx.ist.psu.edu/viewdoc/download?doi=10.1.1.458.7746\&rep=rep1\&type=pd f

Ley que Crea el plan de formación ciudadana para los establecimientos educacionales reconocidos por el estado N²0.911. Recuperado de https://www.leychile.cl/Navegar?idNorma=1088963

Ministerio de Educación de Chile. (2012a). Bases curriculares de la educación chilena, Santiago, Chile: Ministerio de Educación.

Ministerio de Educación de Chile. (2012b). Estándares orientadores para carreras de pedagogía en educación media. Santiago, Chile: Ministerio de Educación.

Niemi, Richard y Junn, Jane. (1998). Civic education: what makes students learn. Yale, Estados Unidos: Yale University Press.

Organización de las Naciones Unidas para la Educación, la Ciencia y la Cultura. (Ed.). (2017). La formación inicial Docente en educación para la ciudadanía en América Latina análisis comparado de seis casos nacionales. Paris, Francia: Autor

Prats, Enric. (2012). ¿Educación cívica o educación para la ciudadanía? Lo que acontece en Europa. En III Congreso Nacional de Educación Comparada. Sociedad Española de Educación Comparada. Universidad de Huelva. Huelva: España.

Quiroz, Ruth y Jaramillo, Orlanda. (2009). Formación ciudadana y educación cívica ¿cuestión de actualidad o de resignificación?. Revista de Teoría y Didáctica de las Ciencias 
Sociales, 14, 123-138. Recuperado de https://dialnet.unirioja.es/servlet/articulo?codigo=3159036

Schugurensky, Daniel y Myers, John. (2003). Learning to teach citizenship: a lifelong learning approach. Journal of the Theory and History of Education International Research Group, 4, 145-166. Recuperado de https://pdfs.semanticscholar.org/c0ad/fa23507aa6554b503a089bbe4f665dc3ba85.pdf

Shulman, L. (2005). Conocimiento y enseñanza: fundamentos de la nueva reforma. Profesorado. Revista de currículum y formación del profesorado, 9(2), 1-30. Recuperado de http://www.redalyc.org/articulo.oa?id=56790202

Schulz, Wolfram, Ainley John, Cox Cristián y Friedman, Tim (2016). Percepciones de los jóvenes acerca del gobierno, la convivencia pacífica y la diversidad en cinco países de América Latina Estudio Internacional sobre Educación Cívica y Ciudadana 2016 de la IEA Informe Lationamericano. Amsterdam, Holanda: Asociación Internacional para la Evaluación del Logro Educativo.

Schulz, Wolfram, Ainley, John, Friedman, Tim y Lietz, Petra (2009). Informe latinoamericano del ICCS 2009: Actitudes y conocimientos cívicos de estudiantes de secundaria en seis países de América Latina. Amsterdam, Holanda: Asociación Internacional para la Evaluación del Logro Educativo.

Velásquez, Elda, Martínez, Loreto, y Cumsille, Patricio. (2014). Expectativas de autoeficacia y actitud prosocial asociadas a participación ciudadana en jóvenes. Psykhe, 13(2), 8598. doi: 10.4067/S0718-22282004000200007 
Revista indizada en

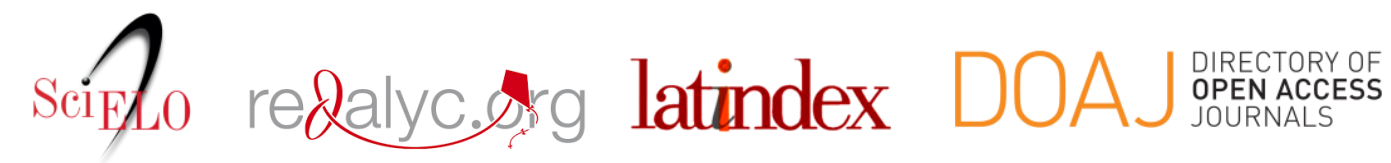

Distribuida en las bases de datos:

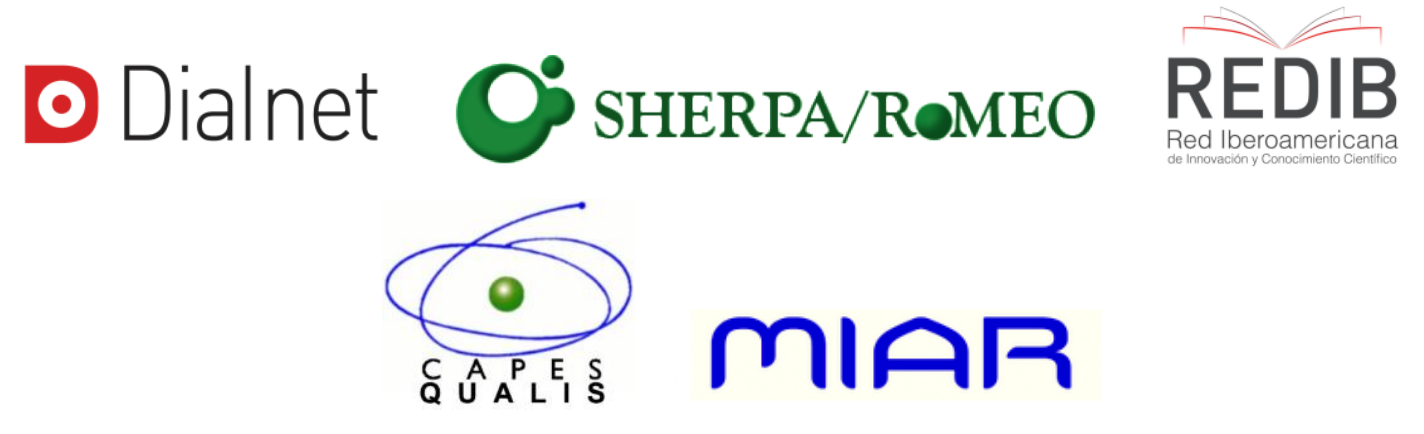

